\title{
Conventional and Alternative Measures for IBS Management
}

\section{Abdul Kader*}

Secretary and Treasurer, Dr. M. Nasirullah Memorial Trust, Tejgaon, Dhaka, Bangladesh

*Corresponding Author: M Abdul Kader, Secretary and Treasurer, Dr. M. Nasirullah Memorial Trust, Tejgaon, Dhaka, Bangladesh.

Received: September 16, 2019; Published: October 14, 2019

DOI: $10.31080 /$ ASGIS.2019.02.0088

\section{Abstract}

Irritable bowel syndrome (IBS) or irritable bowel disease (IBD), is also known as spastic colitis, mucus colitis, and nervous colon. It is a chronic, or long-term, condition, but symptoms tend to change over the years. It's not uncommon for people with IBS to have episodes of both constipation and diarrhea. Symptoms such as bloating and gas typically go away after a bowel movement. There is no cure for IBS. Treatment is aimed at symptom relief. Certain foods as well as stress and anxiety can be triggers for IBS symptoms for many people. Medications are available to ease the symptoms of IBS, but some patients feel better trying natural remedies instead of (or in addition to) conventional drugs.
\end{abstract}

Keywords: Irritable Bowel Syndrome; Low-FODMAP; Cognitive Behavioral Therapy; Complementary and Alternative Medicine; Chinese Herbal Medicine; Brain-Gut Axis

\section{Abbreviation}

IBS: Irritable Bowel Syndrome; CBT-IE: Interoceptive Exposurebased CBT Program; FODMAP: Fermentable Oligo-, Di-, Monosaccharides And Polyols; CAM: Complementary and Alternative Medicine.

\section{Introduction}

About $30 \%$ to $40 \%$ of adults claim to have frequent indigestion, and over 50 million visits are made annually to ambulatory care facilities for symptoms related to the digestive system. IBS is present in patients with symptoms of chronic abdominal pain and altered bowel habits but no identifiable organic etiology. IBS has a prevalence of $1 \%$ to $20 \%$ worldwide, although up to $75 \%$ affected individuals never seek care. Diagnosing IBS can be challenging due to the nonspecific nature of symptoms, overlapping upper and lower abdominal symptoms, and the frequent presence of somatic and psychological comorbidities. Up to $80 \%$ of IBS patients identify food as a possible trigger for their symptoms, so they increasingly ask for dietary and behavioral counseling. Moderate-severe IBS is estimated to account for around $60 \%$ of all IBS cases and has been shown to impose a considerable burden on patients. It is estimated that IBS-C accounts for around $30 \%$ of IBS cases. The economic burden of IBS in the US is estimated at $\$ 28$ billion annually, a portion of these costs may be related to unnecessary and high-frequency tests, although few studies have assessed the factors underlying frequent tests and procedures among patients with IBS. $32 \%$ of IBS-C patients suffer depression as their condition almost every day in the previous month. If main IBS symptom is constipation, linaclotide and lubiprostone are two drugs that are recommended by the American College of Gastroenterology (ACG). Sexual dysfunction is positively associated with perceived GI symptom severity and HRQoL.

\section{Conventional treatments}

Due to disappointing results with conventional IBS treatments, complementary and alternative medicines are becoming attractive options for many patients. Up to $50 \%$ of patients declared that they used some form of CAM for their GI symptoms, ranging from biologically active compounds to mind-body interventions. CAM alone and in conjunction with pharmacological treatments as an integrative approach to manage patients with IBS and improve their QoL. Prokinetics are not specific to IBS and increase gastrointestinal motility in general by acting via dopamine and 5-HT3 receptors as antagonists or 5-HT4 receptors as agonists [2]. Along with prokinetics, treatment revolves around the use of therapies which are not specifically approved/not truly effective for treating IBS-C, such as laxatives, antispasmodics, gastric relaxants, or central neuromodulators and bulking agents (e.g. dietary fibers) [3]. Novartis has agreed to continue to supply Zelnorm ${ }^{\circledR}$ (Tegaserod maleate) for use in emergency situations, due to an increased cardiovascular risk [4]. Alosetron hydrochloride (Lotronex), voluntarily withdrawn in November 2000 by GlaxoSmithKline, but put back on the market, is the only medication approved for the treatment of severe IBS-D in women who have inadequately responded to conventional therapy [5]. However, no studies have evaluated the efficacy of alosetron using the new FDA composite endpoint which requires improvement in both abdominal pain and diarrhea [6]. Fecal calprotectin, an indicator of colonic inflammation, is associated with non-con- 


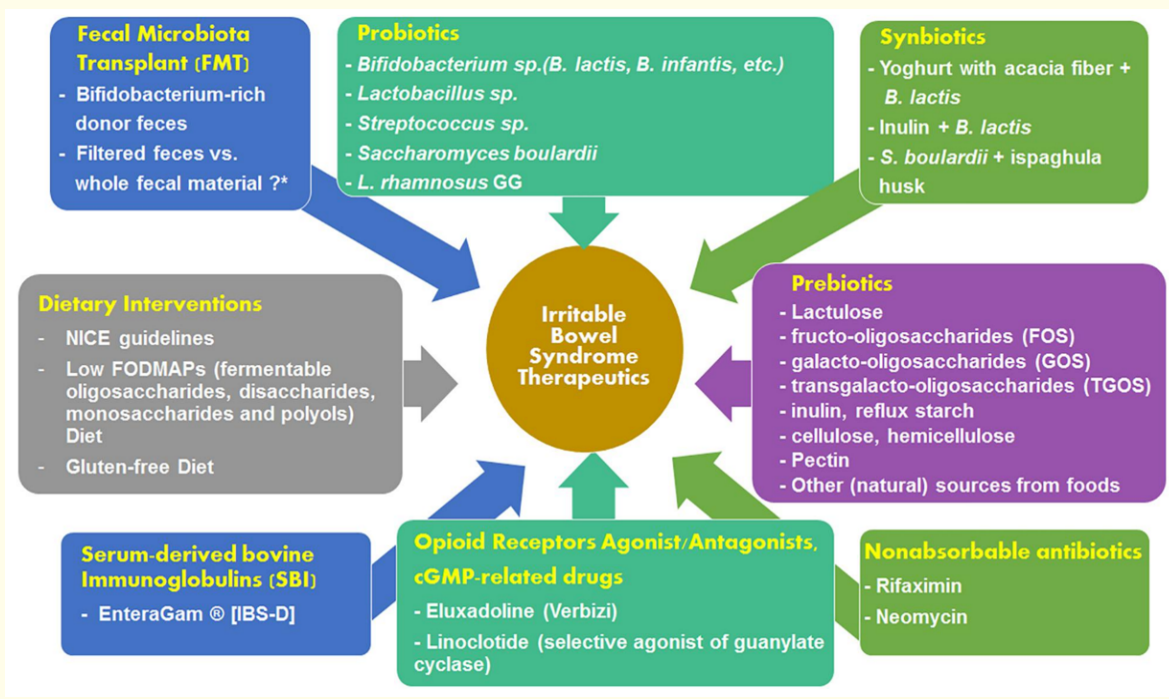

Figure 1: Summary of the available treatment options for mitigating the severity of IBS symptoms [1]. Major demerit of probiotics is that most have a short lifespan and thus repeated doses are necessary. Prebiotics may serve as an alternative treatment as they provide the metabolizable substrates for growth of specific bacteria and hence can alter the microbiota. Xifaxan ${ }^{\circledR}$ was approved by the US FDA in 2015 for the treatment of IBS-D in adults. EnteraGam ${ }^{\circledR}$, is also approved by the US FDA for use in patients with chronic loose and frequent stools including IBS-D and IBD under physician supervision.

stipated IBS. It can be a useful biomarker for measuring the effect of rifaximin therapy. In non-constipated IBS without documented small intestinal bacterial overgrowth (SIBO), Xifaxan ${ }^{\circledR}$ (rifaximin) treatment is associated with acceleration of colonic transit and changes in microbial richness. On the other hand, another antibiotic, neomycin has been shown to improve global IBS symptoms by $50 \%$. Nonetheless, unlike rifaximin, neomycin had adverse effects and induced rapid bacterial resistance or Clostridium difficile infection [7]. Additionally, patients with IBS may experience a range of altered bowel habits, including diarrhea, constipation or alternating constipation and diarrhea. Besides that, digestive symptoms such as dyspepsia, dysphagia, non-cardiac chest pain and nausea are also frequently encountered in patients with IBS. On the other hand, IBS also showed comorbidity with other functional gastrointestinal disorders and association with non-gastrointestinal disorders such as chronic pelvic pain, temporomandibular joint disorder, fibromyalgia and chronic fatigue syndrome. Studies have demonstrated that EnteraGam ${ }^{\circledR}$ is safe and improves GI symptoms (e.g., chronic loose and frequent stools, abdominal discomfort, bloating, and urgency). Approximately $25 \%-50 \%$ of orally administered IgG survives digestion in the stomach and small intestine [8]. Eluxadoline (mixed $\mu$-opioid receptor agonist- $\delta$-opioid receptor antagonist and $\kappa$-opioid receptor agonist) appears safe and effective for treating IBS-D symptoms in patients with an intact gallbladder reporting inadequate relief with prior loperamide use. It has the potential to impact HRQoL in patients with IBS-D via improvement of the burdensome symptoms of IBS-D, including abdominal pain, diarrhea, and urgency [9].

\section{Alternative approaches}

A mixture of dried powdered slippery elm bark, lactulose, oat bran, and licorice root significantly improved both bowel habit and IBS symptoms in patients with IBS-C [10]. More than $95 \%$ of patients rated artichoke leaf extract as better than or at least equal to previous therapies administered for their symptoms, and the tolerability was very good [11]. Probiotics may be useful in the management of IBS; however, dose and specific bacterial strain are important [12]. Enteric-coated peppermint oil is a safe and effective therapy for the relief of abdominal pain and global symptoms and in adults with IBS. Menthacarin, the primary component blocks $\mathrm{Ca} 2+$ channels and causing the relaxation of intestinal smooth muscle tissue [13]. STW 5 is a liquid formulation of nine herbs (Iberis amara totalis recens, Angelicae radix, Cardui mariae fructus, Chelidonii herba, Liquiritiae radix, Matricariae flos, Melissae folium, Carvi fructus and Menthae piperitae folium) used in clinical practice in Germany for more than 50 years, acts beneficially on abdominal symptom clusters as well as on individual GI symptoms in adults and children with efficacy, tolerability, reduced children school absenteeism [14]. Turmeric (Curcuma longa) or Java ginger (Curcuma xanthorrhiza) or curcumin, a biologically active phytochemical or combinatiomn with fennel oil was found to be beneficial, improved patient QoL (due to myorelaxant effect towards the intestinal muscle, involves not only the cholinergic receptors, but also L type Calcium channels) but not statistically significant in IBS symptoms (compared with placebo) [15]. Enzymes comprise the endocannabinoid system in intestinal pain and motility in IBS is also claimed [16] but no significant difference found with dronabinol/nabilone 
(synthetic compounds containing cannabinoids found in the marijuana plant) [17]. Aloe Vera found to be improved QoL with insignificant/no severity symptom reduction in several studies [8794] and studies show its carcinogenic potential in the colon [18], nephrotoxicity and hepatotoxicity [19] which surely demands very limited use unless necessary. Zingier officinale also showed limited potential in symptom management [20]. Mixture of Boswellia carterii, Zingiber officinale and Achillea millefolium improved QoL in men but not in women [21]. Although, an earlier study with Mentha longifolia, Cyperus rotundus and Zingiber officinale combination showed significant improvements after 8-weeks of treatment [22]. CAM for IBS include hypnosis, acupuncture, cognitive behavior therapy, yoga, probiotics, meditation, and herbal medicine [23]. Recent studies reported efficiency and safety of acupuncture alone or combined with Moxibustion or other CHM in IBS [24]. Like Zingier officinale and Curcuma longa, Fumaria officinalis, Hypericum perforatum, Plantago psyllium and Carmint (Mentha spicata, Melissa officinalis, Coriandrum sativum) do not have significant efficacy or at least similar efficacy as placebo is also reported [11]. CHM like Sishen Wan, Ma Zi Ren Wan, CCH1 (patented, modified herb formula), Hemp seed pill, Jianpi Tiaogan Wenshen Recipe (JTWR), Chinese Medicine syndrome-differentiation therapy, Yun-chang capsule, plantain-senna granule (CPSG), Tongxie Yaofang (TXYF) Granule, Changjishu soft elastic capsule, Tongyouqing and many more showed potentials in IBS [25]. The emerging role of brain-gut therapies in IBS are visible. Cognitive behavioral therapy (CBT) and gut-directed hypnosis are the primary behavioral interventions that are introduced to patients with gastrointestinal conditions [26]. IBS involves dysregulation of the brain-gut axis and psychological processes play an important role in the development and maintenance of the disorder [27]. Gut-focused hypnotherapy was found to be effective in primary and secondary care [28], but only small changes were found in intestinal microbiota composition [29]. CBT-IE for IBS includes exposure to abdominal sensations in addition to psychoeducation, self-monitoring, cognitive restructuring, attention training, and in vivo exposure, which are often used in traditional CBT [30]. Home-based version of CBT produced significant and sustained gastrointestinal symptom improvement for patients with IBS compared with education [31]. Both web/phonedelivered CBT and home/clinic-based CBT was found to be effective than usual treatment in refractory IBS, resulted in substantial and enduring relief of multiple symptoms [32,33]. Although significant for both outcomes, the statistical analysis revealed CBT interventions have a greater effect on alleviating IBS symptoms severity rather than on reducing psychological distress [34]. It has recently been found that many patients with IBS have poor dietary habits, with irregular meal intake and high intake of cereals, sweets, and soft drinks, and a low intake of vegetables, fruits, and fish, with correlations between the intake of soft drinks and gastrointestinal (GI) symptoms. According to Britain's National Institute for Health and Care Excellence (NICE) guidelines for dietary and lifestyle advice, dietary and nutritional perspectives should be considered in administering appropriate advice to IBS patients [35]. Emotional stress exacerbates IBS symptoms, and mind-body interventions may be beneficial. Exercise (yoga, walking/aerobic physical activity, Tai Ji, mountaineering, and Baduanjin qigong activity) is potentially a feasible and effective treatment for IBS patients [36]. Yoga improved sleep, increased visceral sensitivity and reduced abdominal pain in teens [37], adolescents and young adults [38], women [39] and postmenopausal women [40]. Patients with IBS might benefit from yoga and a low-FODMAP diet, as both groups showed a reduction in gastrointestinal symptoms [41]. Low-FODMAPs, ketogenic gluten-free diets are considered therapeutic [42]. However, there are gaps in implementation of the low FODMAP diet in clinical practice, as well as long-term safety and efficacy [43]. A low FODMAP diet is only recommended as a second line treatment guided by qualified clinicians with specialized training [44]. Dietary sources fibers include oats, psyllium, ispaghula, nuts and seeds, some fruit and vegetables and pectins. An increase in fiber has often been suggested as an initial treatment for IBS [45]. There is strong evidence to support three mechanisms of action: a) Augmentation of small intestinal water b). Increased colonic fermentation c). Immune modulation [46]. However, water-insoluble fiber does not improve IBS symptoms, consuming soluble fiber improves overall IBS symptoms [47].

\section{Conclusion}

Current drug options including antispasmodic, antidiarrheals, rifaximin, antidepressants, Laxatives and motility accelerants are limited by barely ideal efficacy or side-effect [48]. CD4 + T-cells from IBS-D patients exhibit immune activation, but this did not appear to correlate with psychological stress measurements or changing symptoms over time. It can be said that CD4+ T-cell cytokines and gut homing reveals immune activation in IBS and is largely confined to IBS-D patients but requires further investigation [49]. Probiotics is a safety choice to improve the overall symptoms for IBS patient. The human gut microbiome is genetically diverse, expressing approximately 150-times more genes than the human host. Since it is agreed that the majority of the bacteria commonly identified in the microbiome cannot be routinely cultured using traditional laboratory techniques ( $80 \%$ or greater) [50]. Probiotic supplements are thought to improve IBS symptoms through manipulation of the gut microbiota, but the exact mechanisms of probiotics in the human body are not fully understood.

\section{Acknowledgement}

I'm thankful to Dr. Elshazaly Saeed F.E.H. Elhassan, King Saud University, Saudi Arabia for his precious time to review my literature and for her thoughtful suggestions. I'm also grateful to seminar library of Faculty of Pharmacy, University of Dhaka and BANSDOC Library, Bangladesh for providing me books, journal and newsletters. 


\section{Bibliography}

1. Chong PP., et al. "The Microbiome and Irritable Bowel Syndrome - A Review on the Pathophysiology, Current Research and Future Therapy". Frontier in Microbiology 10 (2019): 1136.

2. Usher., et al. "Factors Associated With Complementary and Alternative Medicine Use in Irritable Bowel Syndrome: A Literature Review". Psychology, Community and Health 2.3 (2013): 346-361.

3. Bellini M., et al. "Irritable bowel syndrome: a disease still searching for pathogenesis, diagnosis and therapy". World Journal of Gastroenterology 20 (2014): 8807-8820.

4. Basnayake C. "Treatment of irritable bowel syndrome". Australian Prescriber 41 (2018): 145-149.

5. KEY POINT. "Tegaserod withdrawn from U.S. market". APhA Drug (2007).

6. Lacy BE., et al. "Alosetron use in clinical practice: significant improvement in irritable bowel syndrome symptoms evaluated using the US Food and Drug Administration composite endpoint". Therapeutic Advances in Gastroenterology 11 (2018): 1756284818771674.

7. Good L., et al. "New therapeutic option for irritable bowel syndrome: serum-derived bovine immunoglobulin". World Journal of Gastroenterology 21 (2015): 3361-3366.

8. Brenner DM., et al. "Efficacy and Safety of Eluxadoline in Patients With Irritable Bowel Syndrome With Diarrhea Who Report Inadequate Symptom Control With Loperamide: RELIEF Phase 4 Study". American Journal of Gastroenterology 114 (2019): 1502-1511.

9. U.S. Department of Health and Human Services Food and Drug Administration Center for Drug Evaluation and Research (CDER). Guidance for industry: irritable bowel syndrome-clinical evaluation of drugs for treatment.

10. Hawrelak JA and Myers SP. Effects of two natural medicine formulations onirritable bowel syndrome symptoms: a pilot study". Journal of Alternative and Complementary Medicine 16 (2010): 1065-1071.

11. Bahrami HR., et al. "Herbal Medicines for the Management of Irritable Bowel Syndrome: A Systematic Review”. Electron Physician 8 (2016): 2719-2725.

12. National Collaborating Centre for Nursing and Supportive Care (UK). Irritable Bowel Syndrome in Adults: Diagnosis and Management of Irritable Bowel Syndrome in Primary Care [Internet]. London: Royal College of Nursing (UK); 2008 Feb. (NICE Clinical Guidelines, No. 61.) 7, Diet and lifestyle.
13. Alammar N., et al. "The impact of peppermint oil on the irritable bowel syndrome: a meta-analysis of the pooled clinical data”. BMC Complementary Alternative Medicine 19 (2019): 21.

14. Gundermann KJ and Vinson B. "Die funktionelle Dyspepsie bei Kindern-eine retrospektive Studie mit einem Phytopharmakon”. Päd 10 (2004): 1-6.

15. Ng QX., et al. "A Meta-Analysis of the Clinical Use of Curcumin for Irritable Bowel Syndrome (IBS)". Journal of Clinical Medicine 7 (2018). pii: E298.

16. Russo EB. "Cannabinoids in the management of difficult to treat pain". Therapeutics and Clinical Risk Management 4 (2008): 245-259.

17. Wong BS., et al. "Randomized pharmacodynamic and pharmacogenetic trial of dronabinol effects on colon transit in irritable bowel syndrome-diarrhea". Neurogastroenterology and Motility 24 (2012): 358-e169.

18. Guo X and Mei N. "Aloe vera: A review of toxicity and adverse clinical effects'. Journal of Environmental Science and Health 34 (2016): 77-96.

19. Yang HN., et al. "Aloe-induced toxic hepatitis". Journal of Korean Medical Science 25 (2010): 492-495.

20. van Tilburg MA., et al. "Is ginger effective for the treatment of irritable bowel syndrome? A double blind randomized controlled pilot trial". Complementary Therapies in Medicine 22 (2014): 17-20.

21. Kazemian A., et al. "Evaluating the efficacy of mixture of Boswellia carterii, Zingiber officinale, and Achillea millefolium on severity of symptoms, anxiety, and depression in irritable bowel syndrome patients". Journal of Research in Medical Sciences 22 (2017): 120.

22. Sahib AS. Treatment of irritable bowel syndrome using a selected herbal combination of Iraqi folk medicines". Journal of Ethnopharmacology 148 (2013): 1008-1012.

23. Lichtenstein GR. "Brain-Gut Therapies and Irritable Bowel Syndrome". Gastroenterology and Hepatology (N Y) 14 (2018): 399.

24. Yan J., et al. "Acupuncture plus Chinese Herbal Medicine for Irritable Bowel Syndrome with Diarrhea: A Systematic Review and Meta-Analysis". Evidence-Based Complementary and Alternative Medicine (2019): 7680963.

25. Teschke R., et al. "Herbal traditional Chinese medicine and its evidence base in gastrointestinal disorders". World Journal of Gastroenterology 21 (2015): 4466-4490. 
26. Riehl ME. "The Emerging Role of Brain-Gut Therapies for Irritable Bowel Syndrome". Gastroenterology and Hepatology (N Y) 14 (2018): 436-438.

27. Kinsinger SW. "Cognitive-behavioral therapy for patients with irritable bowel syndrome: current insights". Psychology Research and Behavior Management 10 (2017): 231-237.

28. Flik CE., et al. "Efficacy of individual and group hypnotherapy in irritable bowel syndrome (IMAGINE): a multicentre randomised controlled trial". The Lancet Gastroenterology and Hepatology 4 (2019): 20-31.

29. Peter J., et al. "Intestinal Microbiome in Irritable Bowel Syndrome before and after Gut-Directed Hypnotherapy". International Journal of Molecular Sciences 19 (2018). pii: E3619.

30. Kawanishi H., et al. "Cognitive behavioral therapy with interoceptive exposure and complementary video materials for irritable bowel syndrome (IBS): protocol for a multicenter randomized controlled trial in Japan". BioPsychoSocial Medicine 13 (2019): 14.

31. Lackner JM., et al. "Improvement in Gastrointestinal Symptoms After Cognitive Behavior Therapy for Refractory Irritable Bowel Syndrome". Gastroenterology 155 (2018): 47-57.

32. Lackner JM., et al. "Durability and Decay of Treatment Benefit of Cognitive Behavioral Therapy for Irritable Bowel Syndrome: 12-Month Follow-Up". American Journal of Gastroenterology 114 (2019): 330-338.

33. Everitt HA., et al. "Assessing telephone-delivered cognitivebehavioural therapy (CBT) and web-delivered CBT versus treatment as usual in irritable bowel syndrome (ACTIB): a multicentre randomised trial". Gut 68 (2019): 1613-1623.

34. Radu M., et al. "Predictors of outcome in cognitive and behavioural interventions for irritable bowel syndrome. A metaanalysis". Journal of Gastrointestinal and Liver Diseases 27 (2018): 257-263.

35. Nilholm C., et al. "A Dietary Intervention with Reduction of Starch and Sucrose Leads to Reduced Gastrointestinal and Extra-Intestinal Symptoms in IBS Patients". Nutrients 11 (2019). pii: E1662.

36. Zhou C., et al. "Exercise therapy of patients with irritable bowel syndrome: A systematic review of randomized controlled trials". Neurogastroenterology and Motility 31 (2019): e13461.

37. Evans S., et al. "Yoga for Teens With Irritable Bowel Syndrome: Results From a Mixed-Methods Pilot Study". Holistic Nursing Practice 32 (2018): 253-260.
38. Evans S., et al. "Iyengar yoga for adolescents and young adults with irritable bowel syndrome". Journal of Pediatric Gastroenterology and Nutrition 59 (2014): 244-253.

39. Patel N and Lacy B. "Does Yoga Help Patients With Irritable Bowel Syndrome?" Clinical Gastroenterology and Hepatology 14 (2016): 1732-1734.

40. Cramer H., et al. "Characteristics of randomized controlled trials of yoga: a bibliometric analysis". BMC Complementary and Alternative Medicine 14 (2014): 328.

41. Schumann D., et al. "Randomised clinical trial: yoga vs a lowFODMAP diet in patients with irritable bowel syndrome". Alimentary Pharmacology and Therapeutics 47 (2018): 203-211.

42. Reddel S., et al. "The Impact of Low-FODMAPs, Gluten-Free, and Ketogenic Diets on Gut Microbiota Modulation in Pathological Conditions". Nutrients 11 (2019). pii: E373.

43. Mitchell H., et al. "Review article: implementation of a diet low in FODMAPs for patients with irritable bowel syndromedirections for future research". Alimentary Pharmacology and Therapeutics 49 (2019): 124-139.

44. Ooi SL., et al. "Probiotics, prebiotics, and low FODMAP diet for irritable bowel syndrome - What is the current evidence?" Complementary Therapies in Medicine 43 (2019): 73-80.

45. National Collaborating Centre for Nursing and Supportive Care (UK). Irritable Bowel Syndrome in Adults: Diagnosis and Management of Irritable Bowel Syndrome in Primary Care [Internet]. London: Royal College of Nursing (UK); 2008 Feb. (NICE Clinical Guidelines, No. 61.) 7, Diet and lifestyle.

46. Jankovich E and S Watkins. "The Low FODMAP Diet Reduced Symptoms in a Patient With Endometriosis and IBS". South African Journal of Clinical Nutrition 30.4 (2017): 32-36.

47. El-Salhy M., et al. "Dietary fiber in irritable bowel syndrome (Review)". International Journal of Molecular Medicine 40 (2017): 607-613.

48. Nasser Y., et al. "Activation of Peripheral Blood CD4+ T-Cells in IBS is not Associated with Gastrointestinal or Psychological Symptoms". Science Report 9 (2019): 3710.

49. Liang D., et al. "Efficacy of different probiotic protocols in irritable bowel syndrome: A network meta-analysis". Medicine (Baltimore) 98 (2019): e16068.

50. Day RL., et al. "Probiotics: current landscape and future horizons”. Future Science OA 5 (2019): FSO391.

\section{Volume 2 Issue 9 November 2019}

(c) All rights are reserved by $M$ Abdul Kader. 